\title{
Topological mechanics from supersymmetry
}

\author{
Jan Attig, ${ }^{1}$ Krishanu Roychowdhury, ${ }^{2,3}$ Michael J. Lawler, ${ }^{2,4}$ and Simon Trebst ${ }^{1}$ \\ ${ }^{1}$ Institute for Theoretical Physics, University of Cologne, D-50937 Cologne, Germany \\ ${ }^{2}$ Laboratory of Atomic and Solid State Physics, Cornell University, Ithaca, New York 14853, USA \\ ${ }^{3}$ Department of Physics, Stockholm University, SE-106 91 Stockholm, Sweden \\ ${ }^{4}$ Department of Physics, Binghamton University, Binghamton, New York 13902, USA
}

(Received 5 October 2018; published 30 December 2019)

\begin{abstract}
In topological mechanics, the identification of a mechanical system's rigidity matrix with an electronic tightbinding model allows one to infer topological properties of the mechanical system, such as the occurrence of "floppy" boundary modes, from the associated electronic band structure. Here, we introduce an approach to systematically construct topological mechanical systems by an exact supersymmetry (SUSY) that relates the bosonic (mechanical) and fermionic (e.g., electronic) degrees of freedom. As examples we discuss mechanical analogs of the Kitaev honeycomb model and of a second-order topological insulator with floppy corner modes. Our SUSY construction naturally defines a set of topological invariants for bosonic (mechanical) systems, such as bosonic Wilson loop operators that are formulated in terms of a SUSY-related fermionic Berry curvature.
\end{abstract}

DOI: 10.1103/PhysRevResearch.1.032047

In quantum mechanics, supersymmetry (SUSY) explicitly relates bosonic and fermionic degrees of freedom-a fundamental concept that has first been introduced [1-3] in highenergy physics and widely been adopted in the formulation of extensions of the standard model [4]. For nonrelativistic settings, the concept of SUSY has been exploited extensively in the study of random phenomena and quantum chaos in mesoscopic systems [5], where a supersymmetric combination of commuting and anticommuting variables allows for the otherwise intractable calculation of disorder averages. The discovery of topological (classical) mechanics has produced another intriguing setting where two fundamentally distinct degrees of freedom are found to be closely related-the mechanical modes of a classical system are cast in analogy to the wave function of an electronic system [6]. At its heart, this analogy identifies the Newtonian equation governing the classical system, $\ddot{x}=-\mathbf{D} x$, with a Schrödinger equation,

$$
i \frac{\partial}{\partial t}\left(\begin{array}{c}
\sqrt{\mathbf{D}}^{T} x \\
i \dot{x}
\end{array}\right)=\left(\begin{array}{cc}
0 & \sqrt{\mathbf{D}}^{T} \\
\sqrt{\mathbf{D}} & 0
\end{array}\right)\left(\begin{array}{c}
\sqrt{\mathbf{D}}^{T} x \\
i \dot{x}
\end{array}\right)
$$

where the dynamical matrix $\mathbf{D}$ of the original classical system enters the Hamiltonian matrix of the quantum system. Exploiting such matrix analogies has produced some farreaching insight, most prominently in the realization that zeroenergy "floppy" boundary modes in isostatic lattices [7] can be identified with the inherently protected boundary modes of topological insulators [8]. It has previously been noted $[6,8]$ that the Hamiltonian matrix in (1) corresponds to a certain symmetry class (BDI) [9] and therefore generically

Published by the American Physical Society under the terms of the Creative Commons Attribution 4.0 International license. Further distribution of this work must maintain attribution to the author(s) and the published article's title, journal citation, and DOI. has a supersymmetric form [5]. In going one step further, the concept of supersymmetry can be used to explicitly connect the degrees of freedom of a constrained mechanical system such as a balls and springs model or certain frustrated magnets with complementary fermionic degrees of freedom and so inherit the topological properties of these fermions [10]. More precisely, considering that the mechanical system is described in terms of real-space coordinates and momenta $(q, p)$, which are both real bosonic variables, their natural SUSY partners are not complex fermions but real fermions, i.e., Majorana fermions.

Here, we report our discovery that we can also use SUSY to map fermionic systems to bosonic ones while preserving locality in both and give a recipe for how to fabricate these bosonic systems with metamaterials. As examples of this construction, we discuss mechanical incarnations of the $Z_{2}$ spin-liquid phase in the Kitaev honeycomb model and of a second-order topological insulator. Furthermore, this SUSY construction allows us to identify topological properties of the bosonic (mechanical) system by explicitly associating it with a fermionic Berry curvature. We showcase such a calculation by evaluating bulk topological invariants, i.e., bosonic Wilson loops, for the mechanical equivalent of a second-order topological insulator. Our results demonstrate that one can construct different topological mechanical systems by using the concept of supersymmetry to directly relate many of the recent advances from the field of topological quantum matter to mechanical analogs.

SUSY construction. To set the scene for our SUSY construction of topological mechanical systems, let us briefly recapitulate the concept of supersymmetry. The central object here is a non-Hermitian SUSY charge operator

$$
\mathcal{Q}=c_{i}^{\dagger} \mathbf{R}_{i j} b_{j}
$$

that connects the annihilation operator of a (complex) boson $b$ with the creation operator of a (complex) fermion $c$ via 
an arbitrary matrix $\mathbf{R}$. The indices $i$ and $j$ run over the total number of fermionic and bosonic degrees of freedom, respectively. From this charge operator one can immediately construct a supersymmetric Hamiltonian

$$
\mathcal{H}_{\mathrm{SUSY}}=\left\{\mathcal{Q}, \mathcal{Q}^{\dagger}\right\}=c^{\dagger} \mathbf{R} \mathbf{R}^{\dagger} c+b^{\dagger} \mathbf{R}^{\dagger} \mathbf{R} b
$$

that decomposes into decoupled bosonic and fermionic parts. By construction, these two partner Hamiltonians are not only both quadratic and isospectral to one another, but their eigenstates are explicitly related by $\mathcal{Q}$, allowing a one-to-one identification of bosonic and fermionic states [11].

For the case of topological mechanics, with phase-space coordinates $(q, p)$ for bosonic degrees of freedom, we have to further specialize this SUSY connection to the case of real bosons and real fermions (and later take the classical limit). More explicitly, we are now led to consider a Hermitian SUSY charge

$$
\mathcal{Q}=\gamma_{i}^{B} \mathbf{1}_{i j} \hat{p}_{j}+\gamma_{i}^{A} \mathbf{A}_{i j} \hat{q}_{j}
$$

that connects the two bosonic degrees of freedom $(\hat{p}, \hat{q})$ (where the hats indicate that these are still quantum mechanical operators fulfilling the usual commutator relations $\left[\hat{q}_{i}, \hat{p}_{j}\right]=i \delta_{i, j}$, with a matching number of two species of Majorana fermions $\gamma^{A}$ and $\gamma^{B}$. Note that in comparison to the complex boson/fermion case of Eq. (2) we have restricted the matrix $\mathbf{R}$ to a block-diagonal form $\mathbf{R}=\left(\begin{array}{ll}1 & \mathbf{0} \\ \mathbf{0} & \mathbf{A}\end{array}\right)$ for reasons that are apparent when looking at the SUSY Hamiltonian $H_{\text {SUSY }}=\left\{\mathcal{Q}, \mathcal{Q}^{\dagger}\right\}$ that again decomposes into bosonic and fermionic partner Hamiltonians, taking the form

$$
\begin{gathered}
\mathcal{H}_{\text {fermion }}=i \gamma_{j}^{B} \mathbf{A}_{j k}^{T} \gamma_{k}^{A}-i \gamma_{j}^{A} \mathbf{A}_{j k} \gamma_{k}^{B}, \\
\mathcal{H}_{\text {boson }}=\hat{p}_{i} \hat{p}_{i}+\hat{q}_{i}\left(\mathbf{A}^{T} \mathbf{A}\right)_{i j} \hat{q}_{j} .
\end{gathered}
$$

Written in this way, the Majorana Hamiltonian (5) describes the hopping of Majorana fermions between two types of sites $A$ and $B$, as it is realized, for instance, for nearestneighbor hopping on bipartite lattices. In such a two-sublattice realization, the bosonic operators reside only on one of the sublattices (namely, $B$ ).

Taking the classical limit of the bosonic Hamiltonian (6), one can further read off that in this form $\mathbf{R}$ in fact corresponds to the rigidity matrix of the mechanical system, with its upper left block giving rise to the mass matrix (set to unity here) and the lower right block giving rise to the dynamical matrix via $\mathbf{D}=\mathbf{A}^{T} \mathbf{A}$. To summarize these steps, we have accomplished that by restricting the matrix $\mathbf{R}$ in the SUSY charge for the real boson/fermion case (4) it may not only be interpreted as the rigidity matrix of the classical system, but it also connects the mechanical system to a particularly accessible form of Majorana hopping problems. In more practical terms, our particular choice of $\mathbf{R}$ allows us to directly connect a number of well-known Majorana systems [of form (5)] to mechanical analogs as given by Eq. (6).

Finally, we note that time-reversal symmetry is preserved in the supersymmetry construction [12]. In short, time-reversal symmetry $\mathcal{T}$ transforms under SUSY as $\mathcal{T}_{b}=$ $\mathbf{R}^{-1} \mathcal{T}_{f} \mathbf{R}=\mathcal{T}_{f}$, because $\left[\mathbf{R}, \mathcal{T}_{f}\right]=\mathbf{0}$ (where $b$ and $f$ refer to bosons and fermions, respectively). As a consequence, any (topological) features of the fermion system protected by time-reversal symmetry will be protected by both timereversal and supersymmetry in the bosonic system.

Classical balls and springs models. The final step in our SUSY construction is to formulate the classical limit of the real boson Hamiltonian (6) as an easy-to-engineer classical model, a system of balls and springs whose Hamiltonian can be written as

$$
\begin{aligned}
\mathcal{H} & =\sum_{i} \frac{p_{i}^{2}}{2 m}+\sum_{i j} \frac{k_{i j}}{2}\left(q_{i}-q_{j}\right)^{2}+\sum_{i} \frac{\kappa_{i}}{2} q_{i}^{2} \\
& \sim \sum_{i} p_{i}^{2}+\sum_{i j} q_{i} \mathbf{D}_{i j} q_{j},
\end{aligned}
$$

where the spring constants $k_{i j}$ and $\kappa_{i}$ can be extracted from the dynamical (spring) matrix $\mathbf{D}=\mathbf{A}^{T} \mathbf{A}$ as

$$
\begin{gathered}
k_{i j}=-2 \sum_{a \in A} \mathbf{A}_{i a}^{T} \mathbf{A}_{a j}, \\
\kappa_{i}=2 \sum_{a \in A} \mathbf{A}_{i a}^{2}-\sum_{b \in B} k_{i b} .
\end{gathered}
$$

The intersite spring constants $k_{i j}$ are the off-diagonal elements of D, that by virtue of our SUSY construction arise from next-nearest-neighbor Majorana hopping (within the boson sublattice $B$ ) [13]. The on-site spring constants $\kappa_{i}$ are the diagonal elements of $\mathbf{A}^{T} \mathbf{A}$ arising from Majoranas hopping back and forth, modified by a contribution coming from the intersite springs.

Mechanical Kitaev model. We now proceed to discuss a classical analog of the celebrated Kitaev model [14], a spin model with characteristic bond-directional exchanges on the honeycomb lattice. The analytical solution of this model [14] is achieved by recasting it in terms of noninteracting Majorana fermions hopping on the same honeycomb lattice [in the background of a classical (static) $Z_{2}$ gauge field] - precisely the type of Majorana Hamiltonian (5) that is amenable to our SUSY construction. Going through the steps outlined above, we end up with a classical balls and springs model on the triangular lattice [15] (i.e., one of the two sublattices of the honeycomb lattice) as illustrated in Figs. 1(a) and 1(b). Each mass, located at a site of the triangular lattice, is restricted to a movement along an axis perpendicular to the lattice plane, and is connected via two types of springs to both the plane and its neighboring masses.

To illustrate the physics of this mechanical Kitaev model, we have integrated the Hamiltonian equations of motion arising from (7) for a system of $40 \times 40$ masses. By applying a periodic drive of a given frequency $\omega$ to a single mass located at the center of the system, we are able to probe individual eigenmodes of the mechanical model. To do so, we take real-space snapshots of the balls and springs configuration (for drives of different frequencies $\omega$ ), which we subsequently Fourier-transform. This allows us to recover the full energy dispersion of the classical system for a given set of fermionic parameters. Comparing these classical dispersions with their corresponding Majorana fermion band structures we find perfect agreement throughout the phase diagram of Fig. 1(c) as shown in Figs. 1(d) and 1(e) for two particular sets of coupling parameters. In Fig. 1(d) we probe the isotropically coupled Kitaev model and recover the well-known Dirac 
(a)

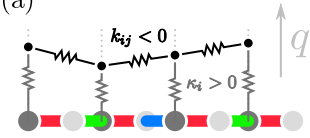

(b)

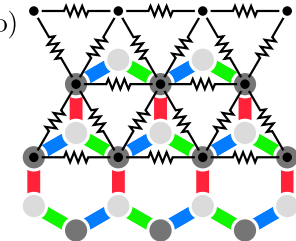

(c)

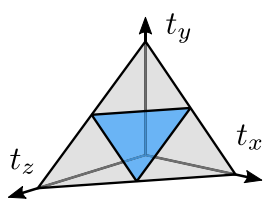

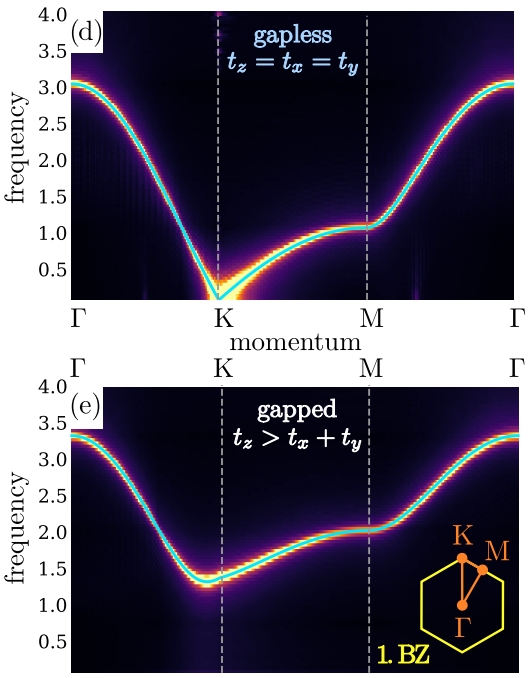

FIG. 1. Mechanical Kitaev model. (a) and (b) Realization in the form of a classical balls and springs model (see also the main text). (c) The phase diagram of the classical model exhibits a gapless region (blue) and three gapped phases (gray). The excitation spectra of the classical model extracted from numerical simulations for the (d) gapless and (e) gapped phases.

cone spectrum of the quantum system. Obtaining such a linear low-energy spectrum in a spring system (which on the level of individual springs always exhibits quadratic energy dispersions) is striking evidence of the many-body physics at play. In Fig. 1(e) we show an energy spectrum for a situation where one of the three coupling parameters dominates and the spectrum exhibits a well-defined low-energy gap, i.e., the mechanical system remains rigid for low-frequency drives up to a threshold given by the gap. While this is imposed from the physics of the quantum system, it is again an unusual situation for a classical system, which typically defies a smallfrequency rigidity (in particular, on the level of individual springs) [16].

The propagating phonon modes constitute the classical analogs of the Majorana fermions in the Kitaev model, with their energy spectra being in one-to-one correspondence. Note that also the underlying $Z_{2}$ gauge structure of the Kitaev spin liquid is fully present in the mechanical model. A pair of gauge excitations - visons in the language of $Z_{2}$ spin liquidscan be excited by flipping the sign of an intersite spring constant [17], in direct analogy to flipping the hopping on a bond in the quantum model. In total, our SUSY construction allows us to build a full mechanical analog of the $Z_{2}$ quantum spin liquid of the Kitaev model, complete with classical analogs of both the fractional quasiparticles (Majorana fermions) and the underlying $Z_{2}$ lattice gauge structure.

Mechanical second-order topological insulator. As a second example, we apply our SUSY construction to derive a classical balls and springs model of the "octupolar insulator" introduced in Refs. [18,19] as a principal example of a second-order topological insulator (SOTI) with topologically protected, gapless corner modes [20]. The original formulation [18] of the SOTI is based on a square-lattice tightbinding model whose hopping strengths are staggered for the elementary square plaquettes of the lattice (which each en- (a)

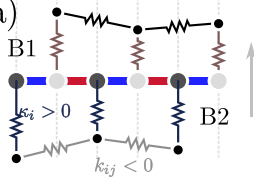

(b)

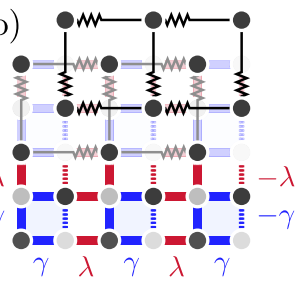

(c)

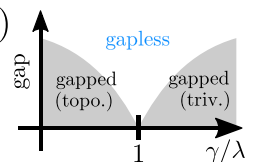

FIG. 2. Balls and springs model of a second-order topological insulator. Mechanical realization shown in (a) side view and (b) top view. As discussed in the main text, the system decouples into two independent systems, denoted here by B1 and B2. (c) Schematic phase diagram for a staggering of the coupling constants around the isotropic coupling point. The excitation spectra of the classical model extracted from numerical simulations for the (d) gapless and (e) gapped (both topological and trivial) phases.

compasses a $\pi$ flux). While the original model is not sensitive to whether the underlying degrees of freedom are complex or real fermions, we again take the real-fermion formulation as the principal input for our SUSY construction. Going through the two steps of first constructing the SUSY-related real boson model (6) and then taking its classical limit (7), we arrive at the balls and springs model illustrated in Figs. 2(a) and 2(b). The mechanical system is composed of two square lattices of coupled balls and springs (denoted B1 and B2 in the figure). The two lattices turn out to be decoupled, since any interlattice coupling always arises from two exchange paths in the quantum model that exactly cancel (as the coupling along the two paths always involves opposite signs as mandated by the plaquette $\pi$ flux in the quantum model). Concentrating on just one of the two mechanical lattices, this leaves us with a system of balls and springs where the masses are again restricted to move along an out-of-plane axis only, with all intersite spring couplings taking the same value (independent of the staggering in the original quantum model). The onsite spring couplings, on the other hand, will be crucial in realizing the topological corner modes of interest here. By construction [Eq. (9)], the on-site couplings are sensitive to the number of neighbors. Considering a system with open boundary conditions, this gives rise to a spatial variation of these couplings along the boundary of the mechanical system.

Probing the mechanical system, we again find a one-to-one correspondence of its bulk energy spectra to the fermionic dispersions for various combinations of $\gamma / \lambda$ as illustrated for two particular staggerings in Figs. 2(d) and 2(e). The occurrence of gapless corner modes in the balls and springs system, predicted by analogy to the quantum model, can be probed by applying a small force to one of the corner 
masses. While in the coupling regime corresponding to the trivial phase of the quantum model there is a restoring force in the mechanical system, this is not the case when entering the coupling regime corresponding to the topological phase in the quantum model (i.e., for $\gamma / \lambda<1$ ). In this regime, the corner mass can be moved arbitrarily far from its original position when exercising an infinitesimal force-this is the gapless corner mode in the mechanical system. That this mechanical corner mode is indeed the signature of a bulk topological phase in the classical system, can be made apparent by considering bulk topological invariants of the mechanical system as discussed in the following.

Topological invariants. A unique aspect of our SUSY construction is that it also defines a way to explore topological properties of mechanical/bosonic systems by connecting the symplectic bosonic eigenfunctions with a fermionic Berry phase of its SUSY partner. The SUSY formalism thereby provides a platform to define bosonic invariants that characterize the topology of an arbitrary mechanical model, specified solely by its rigidity matrix, that go beyond the classification based on conventional bosonic Berry phases (which are not capable of revealing the topological order).

Consider a SUSY pair of fermion and boson models. For the fermionic case, the Berry connection is given by

$$
\mathcal{A}=\left\langle u_{m}(\mathbf{k})\left|i \nabla_{k}\right| u_{n}(\mathbf{k})\right\rangle,
$$

where $\left|u_{m}(\mathbf{k})\right\rangle$ are the eigenvectors of the fermionic Hamiltonian. These eigenstates map to the bosonic eigenstates $\left|v_{m}(\mathbf{k})\right\rangle$ via the rigidity matrix (2)

$$
\left|u_{m}(\mathbf{k})\right\rangle=\frac{\mathbf{R}(\mathbf{k})}{\sqrt{\left|\omega_{m}(\mathbf{k})\right|}}\left|v_{m}(\mathbf{k})\right\rangle \equiv \tilde{\mathbf{R}}(\mathbf{k})\left|v_{m}(\mathbf{k})\right\rangle,
$$

with the prefactor ensuring the symplectic normalization $\left\langle v_{m}(\mathbf{k})\left|\sigma_{2}\right| v_{n}(\mathbf{k})\right\rangle=\left(\sigma_{3}\right)_{m, n}$ of the bosonic eigenproblem [12]. Inserting into (10) then leaves us with a definition of the fermionic Berry curvature in terms of the bosonic states

$$
\begin{aligned}
\mathcal{A}_{\text {SUSY }} & =\left\langle v_{m}(\mathbf{k})\right| i \tilde{\mathbf{R}}^{\dagger} \nabla_{k}\left(\tilde{\mathbf{R}}\left|v_{n}(\mathbf{k})\right\rangle\right) \\
& =\left\langle v_{m}(\mathbf{k})\left|i \sigma_{2}\left(\nabla_{k}+\sigma_{2} \tilde{\mathbf{R}}^{\dagger} \nabla_{k} \tilde{\mathbf{R}}\right)\right| v_{n}(\mathbf{k})\right\rangle .
\end{aligned}
$$

Note that the SUSY construction adds a covariant derivative to the conventional definition of a Berry curvature for the bosonic eigenstates. Importantly, bosonic eigenstates that are trivial with regard to the conventional definition can be revealed as topological states using this augmented definition.

This SUSY framework allows for a comprehensive discussion of the topology of bosonic systems. Applying it to the SOTI balls and springs model, we can demonstrate that its corner modes indeed owe their existence to the topology of the bulk excitation spectrum, shown in Fig. 2. First, we construct edge Hamiltonians using the technique of Wilson loop operators, generalizing the recently developed approach for fermionic SOTIs [18] to the bosonic systems at hand [12]. Computing the Berry phase from the eigenstates of this edge Hamiltonian allows us to distinguish topologically nontrivial phases from trivial ones for the mechanical SOTI model. As illustrated in Fig. 3, a nontrivial topological phase arises for staggering $\gamma / \lambda<1$. Second, a key feature of topological phases is their stability against disorder. We have verified

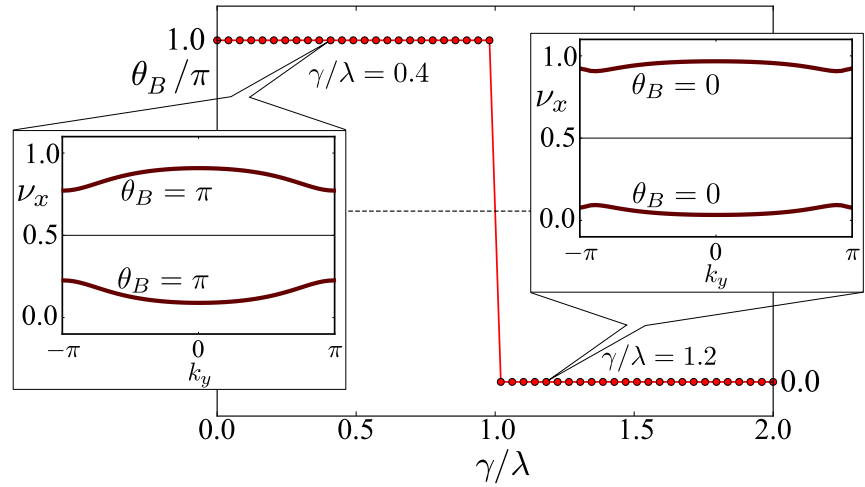

FIG. 3. Bosonic topological invariants. The Berry phase $\theta_{B} / \pi$ of the mechanical SOTI model calculated from the supersymmetric Berry curvature (12) vs the staggering parameters $\gamma / \lambda$. The insets display the eigenvalues $v_{x}$ of the edge Hamiltonian $\mathcal{H}_{x, \mathbf{k}}$ plotted against $k_{y}$ in the topological $(\gamma / \lambda<1)$ and the trivial $(\gamma / \lambda>1)$ phase in which the two bands acquire distinct Berry phases of $\theta_{B}=\pi$ or $\theta_{B}=0$, respectively.

that such a topological robustness is indeed inherited by the mechanical SOTI model, i.e., in the presence of SUSYpreserving disorder topology is indeed preserved, while for SUSY-nonpreserving disorder topological zero modes gap out. For a more detailed discussion of disorder effects, we refer to the Supplemental Material [12]. To conclude, the SUSY framework developed in this Rapid Communication allows us not only to construct bosonic/mechanical equivalents of topological fermion models, but also define other ways of investigating their topological features.

Outlook. The fundamental link between topological mechanics and supersymmetry, which we have explicitly formulated in this Rapid Communication, provides both conceptual and practical insights. On the conceptual side, it is the definition of topological invariants for mechanical (bosonic) systems via the supersymmetric Berry curvature (12), which allows for a different perspective on these systems that might lead to a deeper understanding of the classification of bosonic insulators. In expanding the conceptual connection in the future, it would be interesting to go beyond the single-particle equivalences employed in the current work and use SUSY to connect many-fermion states with mechanical (bosonic) analogs. In practical terms, our SUSY construction allows us to translate many of the topological Majorana band theories to topological balls and springs models ready to be built in the laboratory. As demonstrated for the mechanical Kitaev model and SOTI, the obtained mechanical models might be of intriguing simplicity [21]. One interesting future avenue to explore is whether the mechanical analogs of Majorana fermion systems discussed in this work can regain their quantum mechanical character, e.g., by employing optomechanical systems or nanoscale metamaterials [22], and thereby allow us to realize the bosonic quantum modes that are SUSY partners of Majorana fermions.

Acknowledgments. We thank V. Dwivedi, A. Rosch, and in particular M. Zirnbauer for enlightening discussions. We gratefully acknowledge the hospitality of the Kavli Institute for Theoretical Physics, supported by NSF PHY-1125915, 
where this work was initiated during the "Intertwined orders" program. The Cologne group acknowledges partial support from the Deutsche Forschungsgemeinschaft (DFG, German Research Foundation), Projektnummer 277146847-SFB 1238 (project C02) and Projektnummer 277101999-TRR 183 (project B01). The numerical simulations were performed on the CHEOPS cluster at RRZK Cologne employing the JULIA package DifferentialEquations.jl [23].
[1] Yu. A. Gol'fand and E. P. Likhtman, Extension of the algebra of Poincare group generators and violation of $\mathrm{P}$ invariance, JETP Lett. 13, 323 (1971).

[2] P. Ramond, Dual theory for free fermions, Phys. Rev. D 3, 2415 (1971).

[3] A. Neveu and J. H. Schwarz, Factorizable dual model of pions, Nucl. Phys. B 31, 86 (1971).

[4] H. Baer and X. Tata, The minimal supersymmetric standard model, in Weak Scale Supersymmetry: From Superfields to Scattering Events (Cambridge University Press, Cambridge, UK, 2006).

[5] K. Efetov, Supersymmetry in Disorder and Chaos (Cambridge University Press, Cambridge, UK, 1999).

[6] S. D. Huber, Topological mechanics, Nat. Phys. 12, 621 (2016).

[7] J. C. Maxwell, On the calculation of the equilibrium and stiffness of frames, Philos. Mag. 27, 294 (1864).

[8] C. L. Kane and T. C. Lubensky, Topological boundary modes in isostatic lattices, Nat. Phys. 10, 39 (2013).

[9] A. Altland and M. R. Zirnbauer, Nonstandard symmetry classes in mesoscopic normal-superconducting hybrid structures, Phys. Rev. B 55, 1142 (1997).

[10] M. J. Lawler, Supersymmetry protected topological phases of isostatic lattices and kagome antiferromagnets, Phys. Rev. B 94, 165101 (2016).

[11] More precisely, such a one-to-one identification is possible only for nonzero-energy eigenstates.

[12] See Supplemental Material at http://link.aps.org/supplemental/ 10.1103/PhysRevResearch.1.032047 for more information on mechanical realizations of our spring model, details on corner mode simulations as well as noise in the classical system, time-reversal symmetry, and a detailed derivation of the supersymmetric Berry curvature from the bosonic Wilson loop construction.

[13] For the common case of a uniform sign structure of the Majorana hopping terms, the intersite springs have negative spring constants, while the on-site spring constants are positive.

[14] A. Kitaev, Anyons in an exactly solved model and beyond, Ann. Phys. 321, 2 (2006)

[15] Our model can also be considered to be a particularly simple balls and springs incarnation of "mechanical graphene," which has been first put forward in Ref. [24].

[16] Note that for the system at hand, there are no gapless acoustic phonons, i.e., Goldstone modes arising from the spontaneous breaking of translational symmetry, since our system explicitly breaks this symmetry.

[17] Note that any change to the intersite spring constant also requires a compensating change to the on-site spring constants.

[18] W. A. Benalcazar, B. A. Bernevig, and T. L. Hughes, Quantized electric multipole insulators, Science 357, 61 (2017).
[19] W. A. Benalcazar, B. A. Bernevig, and T. L. Hughes, Electric multipole moments, topological multipole moment pumping, and chiral hinge states in crystalline insulators, Phys. Rev. B 96, 245115 (2017).

[20] Conceptually, SOTIs are part of a family of higher-order topological insulators with protected hinge or corner modes [25] Experimental realizations of SOTIs have been discussed earlier in the context of cleverly engineered phononic insulators [26], microwave systems [27], and electrical circuits [28], along with the observation that bismuth is in fact a SOTI [29]. On a more theoretical basis, SOTIs have also been discussed in the context of frustrated quantum magnetism [30].

[21] However, some of the complexity of the original models might still be hidden in the occurrence of negative spring constants, which might impede a physical realization in the laboratory. We note, however, that negative spring constants do not pose an absolute no-go limit, since they can in fact be realized approximately [12].

[22] M. Aspelmeyer, T. J. Kippenberg, and F. Marquardt, Cavity optomechanics, Rev. Mod. Phys. 86, 1391 (2014).

[23] C. Rackauckas and Q. Nie, DifferentialEquations.jl-A performant and feature-rich ecosystem for solving differential equations in Julia, J. Open Res. Software 5, 15 (2017).

[24] J. E. S. Socolar, T. C. Lubensky, and C. L. Kane, Mechanical graphene, New J. Phys. 19, 025003 (2017).

[25] F. Schindler, A. M. Cook, M. G. Vergniory, Z. Wang, S. S. P. Parkin, B. A. Bernevig, and T. Neupert, Higher-order topological insulators, Sci. Adv. 4, eaat0346 (2018).

[26] M. Serra-Garcia, V. Peri, R. Süsstrunk, O. R. Bilal, T. Larsen, L. G. Villanueva, and S. D. Huber, Observation of a phononic quadrupole topological insulator, Nature (London) 555, 342 (2018).

[27] C. W. Peterson, W. A. Benalcazar, T. L. Hughes, and G. Bahl, A quantized microwave quadrupole insulator with topologically protected corner states, Nature (London) 555, 346 (2018).

[28] S. Imhof, C. Berger, F. Bayer, J. Brehm, L. W. Molenkamp, T. Kiessling, F. Schindler, Ching Hua Lee, M. Greiter, T. Neupert, and R. Thomale, Topolectrical-circuit realization of topological corner modes, Nat. Phys. 14, 925 (2018).

[29] F. Schindler, Zhijun Wang, M. G. Vergniory, A. M. Cook, A. Murani, S. Sengupta, Alik Yu. Kasumov, R. Deblock, S. Jeon, I. Drozdov, H. Bouchiat, S. Guéron, A. Yazdani, B. A. Bernevig, and T. Neupert, Higher-order topology in bismuth, Nat. Phys. 14, 918 (2018).

[30] V. Dwivedi, C. Hickey, T. Eschmann, and S. Trebst, Majorana corner modes in a second-order Kitaev spin liquid, Phys. Rev. B 98, 054432 (2018). 